\title{
Hybrid robotic thoracic surgery for excision of large mediastinal
}

\section{masses}

\author{
Dario Amore, Marcellino Cicalese, Roberto Scaramuzzi, Davide Di Natale, Dino Casazza, Carlo Curcio \\ Division of Thoracic Surgery, Monaldi Hospital, Naples, Italy \\ Contributions: (I) Conception and design: D Amore; (II) Administrative support: D Amore, C Curcio; (III) Provision of study materials or patients: \\ C Curcio, D Amore, M Cicalese; (IV) Collection and assembly of data: D Amore, R Scaramuzzi, D Di Natale, D Casazza; (V) Data analysis \\ and interpretation: D Amore, M Cicalese, R Scaramuzzi, D Di Natale, D Casazza; (VI) Manuscript writing: All authors; (VII) Final approval of \\ manuscript: All authors. \\ Correspondence to: Dario Amore. Division of Thoracic Surgery, Monaldi Hospital, Leonardo Bianchi Street, 1, Naples 80131, Italy. \\ Email: dario.amore@alice.it.
}

\begin{abstract}
Between April 2016 and October 2017, we retrospectively reviewed all patients undergoing excision of large mediastinal masses using a hybrid robotic thoracic approach at the Unit of Thoracic Surgery of Monaldi Hospital in Naples. The inclusion criteria for this approach were: evident unilateral predominance of the mass; absence of invasion to surrounding structures. Planned conversion to sternotomy was necessary in one patient for tenacious adhesions between the mediastinal goiter and the left innominate vein. In all cases the postoperative course was uneventful. The hybrid robotic approach, adopted in our Unit, consists of a thoracic procedure performed completely with articulated surgical instruments under three-dimensional vision and followed by final extension of a port-site incision to retrieve the voluminous specimen. This approach uses all the advantages of robotic technology that enables to perform a fine dissection in the small space of the anterior mediastinum and at the same time, through the simple extension of a minimally invasive access, avoids the painful sequelae of thoracotomy. In selected cases, with increased experience in robotic surgery, it can be proposed for excision of large mediastinal masses, although a longer follow-up period is necessary to validate our findings.
\end{abstract}

Keywords: Hybrid robotic surgery; mediastinum; large masses

Received: 23 April 2018; Accepted: 08 May 2018; Published: 18 May 2018.

doi: 10.21037 /jovs.2018.05.17

View this article at: http://dx.doi.org/10.21037/jovs.2018.05.17

\section{Introduction}

The median sternotomy has been the gold standard for a long time in the surgical management of large mediastinal masses (1) but in the past 20 years a shift has taken place in thoracic surgery, with many resections performed via a video-assisted thoracic surgery (VATS) approach that has resulted in exciting benefits such as reduced length of hospital stay, less trauma and discomfort for the patient (2). However, for delicate maneuvers of dissection in the anterior mediastinum, this approach has limitations due to the reduced maneuverability of the thoracoscopic instruments and the two-dimensional visualization of the operative field. The advent of robotic-assisted technology has allowed to overcome these limitations thanks to the 3-dimensional vision system and the multi-articulated instruments: useful features that ensure greater surgical precision in a narrowly confined and difficult-to-reach anatomic region as the anterior mediastinum (3).

\section{Methods}

At the Unit of Thoracic Surgery of Monaldi Hospital in Naples, between December 2012 and October 2017, a total of 57 patients underwent robotic surgery for treatment of anterior mediastinal diseases. Among those, nine patients (15.8\%) showed large mediastinal masses that were excised 
Table 1 Characteristics of patients undergoing excision of mediastinal masses

\begin{tabular}{lcccl}
\hline Sex & Age & Side approach & Diameters $(\mathrm{cm})$ & Final pathological diagnosis \\
\hline M & 46 & Right & $8 \times 4 \times 3$ & Type B2 thymoma, Masaoka stage I \\
M & 40 & Right & $8.5 \times 8 \times 3$ & Type AB thymoma, Masaoka stage I \\
F & 68 & Right & $11 \times 10 \times 5.4$ & Type AB thymoma, Masaoka stage I \\
F & 51 & Left & $10 \times 7 \times 4$ & Type A thymoma, Masaoka stage I \\
M & 46 & Left & $6 \times 5 \times 2.5$ & Solitary fibrous tumor \\
F & 74 & Left & $7 \times 5 \times 2$ & Multinodular goiter \\
F & 73 & Left & $7 \times 6 \times 3$ & Multinodular goiter \\
F & 61 & Right & $10 \times 6 \times 2$ & Multinodular goiter \\
M & 52 & Right & $11 \times 9 \times 4$ & Omental adipose tissue \\
\hline
\end{tabular}

through a hybrid robotic approach, starting in April 2016. The patient characteristics are listed in Table 1. We defined as large mediastinal mass a specimen with maximum diameter equal to or greater than $6 \mathrm{~cm}$. In this subgroup of patients, consisting of four males and five females with a mean age of 57 years, only common symptoms were experienced as dyspnea, chest pain and no one was suffering from myasthenia gravis. Preoperative work-up included computed tomography (CT), fluorodeoxyglucose positron emission tomography (FGG-PET) and, in some instances, magnetic resonance imaging (MRI) and thyroid scintigraphy with iodine $131\left(\mathrm{I}^{131}\right)$. All procedures were performed under general anesthesia and one-lung ventilation was achieved by use of a double-lumen endotracheal tube. In all cases a transthoracic unilateral three-trocar approach from either left or right side was adopted, placing the patient in incomplete $\left(30^{\circ}-45^{\circ}\right)$ lateral decubitus with the approached side up. A right-sided 3-trocar procedure was performed in five patients $(55.6 \%)$. In all cases, endopleural insufflation of $\mathrm{CO}_{2}$ gas $(6-10 \mathrm{mmHg})$ was imperative to enlarge the space of the operative field. In two patients an additional median cervicotomy incision was made for excision of antero mediastinal retrosternal goiter. At the end of the procedure the specimen was then removed by enlarging one of the port incisions and using an endo-bag (Figure 1). A 28 Fr chest tube was placed in pleural cavity with closure of the thoracic incisions, after reinflation of lung.

\section{Results}

In eight patients (88.9\%), the procedure was completed by hybrid robotic surgery. In one case, the surgical equipe

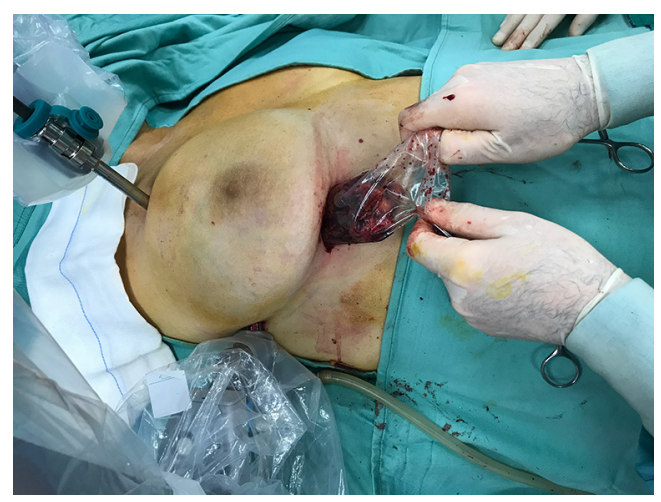

Figure 1 Extraction of the specimen protected by an endobag with enlargement of one of the port incisions.

planned to switch to trans-sternal approach due to severe tenacious adhesions between the mediastinal goiter and the left innominate vein. Mean chest drain duration was 3.1 days (range: $2-5$ days) and the mean hospital stay was 4.2 days (range: $3-7$ days). The final pathological diagnoses were the following: thymoma [4], solitary fibrous tumor [1], multinodular goiter [3] and omental adipose tissue [1]. According to the World Health Organization (WHO) classification: 1 thymoma was type A, 2 type $\mathrm{AB}$ and 1 type B2. Pathologic staging revealed Masaoka stage I in all patients with thymoma. The anterior mediastinal mass with the larger size was a thymoma measuring, on the pathological report, $11 \mathrm{~cm} \times 10 \mathrm{~cm} \times 5.4 \mathrm{~cm}$ in diameters. Postoperative course was uneventful in all cases. During follow-up period, which ranged from 6 months to 2 years, no patient presented with recurrence. 


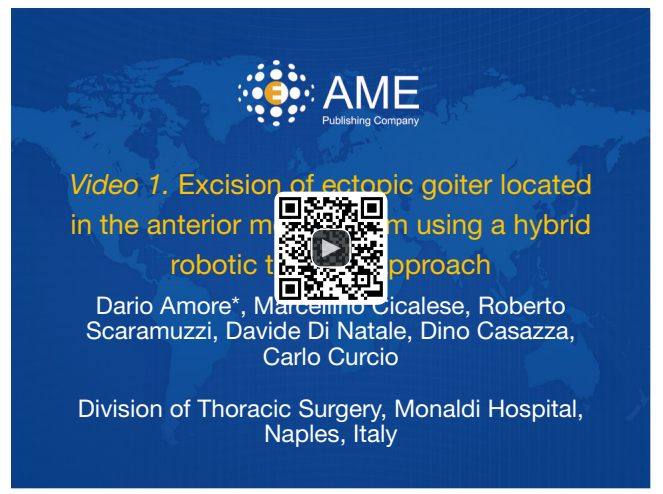

Figure 2 Excision of ectopic goiter located in the anterior mediastinum using a hybrid robotic thoracic approach (6). The video shows how the articulated robotic instruments are able to perform a delicate dissection around vascular structures in a very narrow space. In this confined space, robotic technology allows the surgeon to safely manage even minor bleeding as that coming, in this case, from a brachiocephalic artery branch, divided using the monopolar spatula.

Available online: http://www.asvide.com/article/view/24847

\section{Discussion}

Currently the robotic surgery is widely adopted in the treatment of anterior mediastinal masses thanks to instruments that can articulate with 7 degrees of freedom allowing, through complex three-dimensional movements, a safe dissection around vascular and nervous structures in tiny and difficult-to-reach anatomical regions such as the anterior mediastinum (4,5) (Figure 2). In the literature, however, there is no unanimous agreement on the surgical treatment of large mediastinal masses through minimally invasive techniques, especially for tumors larger than 10 $\mathrm{cm}$, because the manipulation is more difficult $(1,7)$. Some authors have performed resection of bulky intrathoracic benign lesions located in the anterior mediastinum, as benign thymoma or teratoma, via VATS, arguing that many obstacles can be overcome using appropriate instrumentation in careful and skillful hands (8). According to many authors, we argue that the anterior mediastinum is a delicate and difficult to reach anatomic area and that robotic technology, through automatic tremor filtering and articulating instruments, facilitates delicate surgical maneuvers in this region. Therefore, thanks to these technical advantages, robotic surgery system allows to

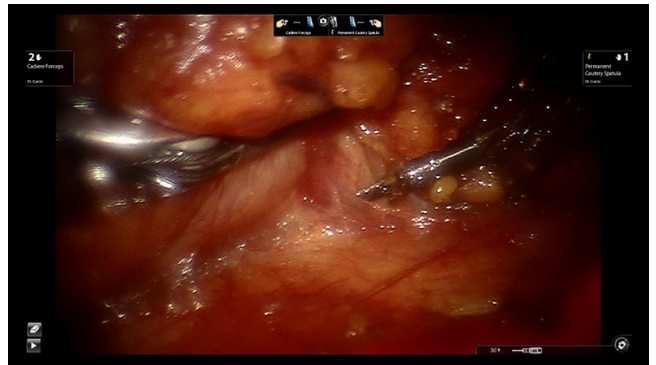

Figure 3 Anterior mediastinal mass lifted by Cadiere forceps while cautery spatula, during dissection, creates a tunnel between the mass and the surrounding structures.

work safely in close proximity to the vulnerable vessels and nerves of the anterior mediastinum (3). Our experience suggests that the robot may also be a useful tool to perform excision of large mediastinal masses with evident unilateral predominance, where CT scans don't show any signs of invasion to surrounding structures. Robotic instruments have 7 degrees of freedom and mimic the articulated movements of the human hand: this feature allows the surgeon, during excision of large mediastinal lesions, to lift the mass and create a dissection plane (Figure 3). This technique, through a stable retraction of the mass, avoids excessive manipulation of surgical instruments and, in this way, reduces the risk of vascular injury during surgical resection. In patients where a giant thymoma is suspected, we always perform extended thymectomy with en bloc resection of the anterior mediastinal fat tissue: using perithymic fat for grasping and traction we thus cut the risk of capsular damage. In the literature, hybrid robotic procedure so far lacks a clear definition. Gharagozloo and co-workers have reported cases of hybrid thoracic surgery consisting of two-phase treatment: robotic hilar and mediastinal dissection, followed by VATS lobectomy (9). Weissenbacher and Bodner have referred to hybrid esophageal procedures in which the robot was used for some steps only (3). In selected patients with large and mainly paramediastinal tumours, some authors have developed a hybrid procedure combining robotic assisted thoracoscopic surgery (RATS) for the mediastinal dissection from one side and a thoracotomy for the tumour resection from the opposite side (10). Our hybrid robotic approach, adopted for the excision of large mediastinal masses and already reported in our single case reports $(11,12)$, consists of a thoracic procedure performed completely under endoscopic 


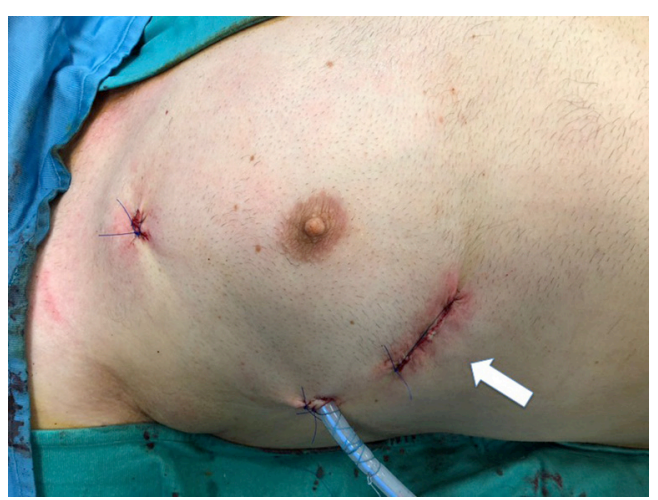

Figure 4 Extension of a port-site incision to pull out the specimen at the end of the procedure (white arrow).

vision, using robotic arms introduced through minimally invasive accesses, and followed by final extension of a portsite incision to retrieve the voluminous specimen (Figure 4). This approach allows to perform delicate maneuvers of dissection within the incredibly narrow space of anterior mediastinum, using the technical advantages of robotic technology; at the same time, through the final extension of a port-site incision for large specimen retrieval, it is less painful than open thoracotomy.

\section{Conclusions}

Although most surgeons agree that a limit of 3 or $5 \mathrm{~cm}$ of diameter of mediastinal masses is considered suitable for minimally invasive approaches (13), we argue that, with increased experience in robotic thoracic surgery, it's possible to perform excision of large mediastinal masses through a hybrid robotic approach. In our report the indications for this procedure have been however limited to selected cases where preoperative work-up, including CT and MRI, showed unilateral mass predominance and a distinct fat plane between the lesion and the surrounding structures. Despite the encouraging results, the effectiveness of our procedure can only be verified by longer term follow-up studies, especially in patients with a stage I thymoma where the mean time to recurrence is 10 years (14).

\section{Acknowledgements}

None.

\section{Footnote}

Conflicts of Interest: The authors have no conflicts of interest to declare.

\section{References}

1. Li WW, van Boven WJ, Annema JT, et al. Management of large mediastinal masses: surgical and anesthesiological considerations. J Thorac Dis 2016;8:E175-84.

2. Toker A, Özyurtkan MO, Kaba E, et al. Da Vinci Robotic System in the surgery for mediastinal bronchogenic cyst: a report on five patients. J Vis Surg 2015;1:23.

3. Weissenbacher A, Bodner J. Robotic surgery of the mediastinum. Thorac Surg Clin 2010;20:331-9.

4. Kuo SW, Huang PM, Lin MW, et al. Robot-assisted thoracic surgery for complex procedures. J Thorac Dis 2017;9:3105-13.

5. Augustin F, Schmid T, Bodner J. Robotic surgery for mediastinal tumors. Eur Surg 2011;43:218-23.

6. Amore D, Cicalese M, Scaramuzzi R, et al. Excision of ectopic goiter located in the anterior mediastinum using a hybrid robotic thoracic approach. Asvide 2018;5:516. Available online: http://www.asvide.com/article/ view/24847

7. Marulli G, Rea F, Melfi F, et al. Robot-aided thoracoscopic thymectomy for early-stage thymoma: a multicenter European study. J Thorac Cardiovasc Surg 2012;144:1125-30.

8. Gossot D, Izquierdo RR, Girard P, et al. Thoracoscopic resection of bulky intrathoracic benign lesions. Eur J Cardiothorac Surg 2007;32:848-51.

9. Gharagozloo F, Margolis M, Tempesta B, et al. Robotassisted lobectomy for early-stage lung cancer: report of 100 consecutive cases. Ann Thorac Surg 2009;88:380-4.

10. Schneiter D, Tomaszek S, Kestenholz P, et al. Minimally invasive resection of thymomas with the da $\mathrm{Vinci}{ }^{\circledR}$ Surgical System. Eur J Cardiothorac Surg 2013;43:288-92.

11. Amore D, Rispoli M, Cicalese M, et al. Anterior mediastinal solitary fibrous tumor resection by da Vinci ${ }^{\circledR}$ Surgical System in obese patient. Int J Surg Case Rep 2017;38:163-5.

12. Amore D, Cicalese M, Scaramuzzi R, et al. Antero mediastinal retrosternal goiter: surgical excision by combined cervical and hybrid robot-assisted approach. J Thorac Dis 2018;10:E199-202.

13. Rea F, Marulli G. Robotic Versus VATS Thymectomy 
for Encapsulated Thymoma. In: Ferguson MK. editor. Difficult Decisions in Thoracic Surgery. Springer, 2014:699-707.

14. Detterbeck FC, Parsons AM. Thymic tumors: a review

Cite this article as: Amore D, Cicalese M, Scaramuzzi R, Di Natale D, Casazza D, Curcio C. Hybrid robotic thoracic surgery for excision of large mediastinal masses. J Vis Surg 2018;4:105.

of current diagnosis, classification and treatment. In: Patterson A, Cooper JD, Deslauriers J, et al. editors. Pearson's Thoracic and Esophageal Surgery 3rd edition. Philadelphia: Churchill Livingstone, 2008:1589-614. 